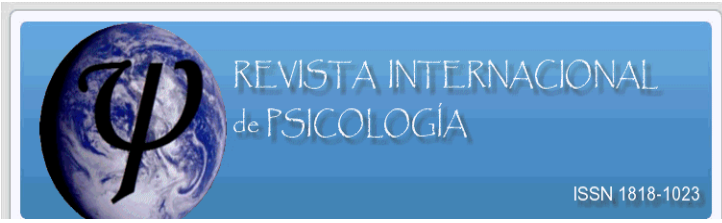

\title{
Percepción y materialización de la valorización social de diferentes conductas obedientes en los obreros argentinos en función de su situación de asalariados o de desocupados
}

\section{Luisa Mayoral $^{1}$, Bernard Gangloff ${ }^{2}$, María del Carmen Romero ${ }^{3}$}

\begin{abstract}
RESUMEN
La valorización social de una conducta y la desvalorización de la conducta opuesta constituyen características típicas de las normas sociales. Se ha constatado, gracias a estudios recientemente realizados en la Provincia de Buenos Aires (Argentina), que los mandos con personal a cargo valorizan las conductas alineadas entre sus subordinados (lo que permite pensar que se está en presencia de una norma social de alineación), y que los subordinados son conscientes de la existencia de esta norma.

Las preguntas que se plantean ahora son las siguientes 1) si los subordinados tienen conciencia de esta norma de manera global o si efectúan diferenciación entre sus diversas materializaciones, 2) si espontáneamente, siguen estas normas en sus diversas materializaciones o actúan de manera selectiva y 3) si estas actitudes globalizadoras o diferenciadoras de sus representaciones y de sus conductas varían en función de la ocupación efectiva o no de un puesto de trabajo.

Para responder a estos interrogantes, hemos utilizado el paradigma de la autopresentación sobrenormativa versus contranormativa. Esto es, hemos presentado a 180 obreros de la Provincia de Buenos Aires (la mitad de ellos con empleo y la otra mitad, sin empleo) una lista de conductas para que ellos indiquen cuáles de ellas adoptarían espontáneamente, cuáles de ellas adoptarían para dar una buena imagen de sí mismos, y cuáles de ellas adoptarían para dar una mala imagen.

Los resultados obtenidos confirman nuestras hipótesis de selectividad en las respuestas y el efecto de la variable empleo.
\end{abstract}

\section{PALABRAS CLAVES}

obediencia, reclutamiento, empleo, desempleo, obreros.

\section{INTRODUCCION}

Toda situación de reclutamiento para un reclutador, puede traducirse en la cuestión siguiente: ¿el candidato posee un buen perfil? Sabemos después de mucho tiempo, que los aspectos esenciales de este perfil involucran más a las competencias sociales que a las competencias técnicas, esto es, el «saber hacer» del candidato. Este saber hacer está constituido por lo que llamamos la personalidad. Hoy por hoy, existe un cuasi-consenso dentro de los psicólogos, en el sentido de considerar que esta personalidad puede describirse gracias a cinco grandes dimensiones, que son : la Energía, la Amabilidad, el Carácter concienzudo, la Estabilidad

\footnotetext{
1 Universidad Nacional del Centro de la Provincia de Buenos Aires, Tandil (Argentina). Correo electrónico: lmayoral@econ.unicen.edu.ar

2 Laboratoire PRIS, Département de Psychologie, Université de Rouen (France) et Département de Postgraduation en Gestion à l'Université Fédérale de Paraïba (Brésil). Courrier électronique: bernard.gangloff@univ-rouen.fr

3 Universidad Nacional del Centro de la Provincia de Buenos Aires, Tandil (Argentina). Correo electrónico: romero@econ.unicen.edu.ar
} 
emocional y la Apertura de espíritu ; es el modelo de los Cinco Grandes ( $c f$. Rolland, 1994, p65; Caprara, Barbaranelli et Borgogni, 1997, p13).

Hemos observado entonces (Gangloff, 2000) que el candidato ideal es generalmente un candidato enérgico (es decir, dinámico y dominante), amable (capaz de prestar cooperación y ser cordial), conscienzudo (es decir, meticuloso y perseverante), emocionalmente estable (capaz de controlar sus emociones y sus impulsos), y de espíritu abierto (abierto a la cultura y a la experiencia).

Por otro lado, recientemente ha surgido otro criterio respecto de este saber hacer, otro factor no ligado a la personalidad, sino de tipo más bien normativo, que ha restado importancia a cada uno de estos cinco factores, y aún a la consideración de la personalidad : la adhesión a la norma de obediencia.

Esta adhesión se traduce en evitar todo tipo de conductas que cuestionen la jerarquía de poderes dentro de un sistema social constituido como tal ( $c f$. para una revisión : Gangloff, 2002). El individuo que adhiera a esta norma será no solamente sumiso a esta jerarquía, sino que también sentirá una suerte de fidelidad, evitando toda conducta, en palabras o actos concretos, que pudieran hacerlo portador de cuestionamientos. En este sentido, los reclutadores prefieren sistemáticamente a un candidato no enérgico, no amable, no concienzudo, emocionalmente inestable o de espíritu cerrado, pero obediente, a otro que posea la personalidad ideal, pero que sea potencialmente rebelde (Gangloff y Huet, 2004). Finalmente, también se ha observado que los candidatos en un reclutamiento, o más globalmente los asalariados, son conscientes de esta preferencia, es decir, son conscientes de la valorización social de la obediencia ( $c f$. Gangloff, 2002).

Si bien la mayoría de los estudios anteriormente citados han sido realizados en Francia, investigaciones complementarias en Argentina arrojan resultados similares. Se ha establecido así que, en las empresas argentinas, los cuadros de mando con responsabilidad jerárquica prefieren trabajar con subordinados obedientes y no con subordinados potencialmente rebeldes. (Gangloff, Mayoral y Duringer, 2005). Asimismo, se ha constatado que los subordinados poseen "clarividencia normativa" respecto de la obediencia (tomando la expresión de Py y Somat, 1991), es decir que son conscientes de la valorización social de la obediencia y que espontáneamente se muestran obedientes antes que rebeldes (Gangloff, Mayoral y Quiroga, 2005).

Sin embargo, la obediencia puede concretarse en diferentes conductas y a raíz de diferentes causas. Así, hemos creído necesario afinar los resultados anteriormente obtenidos, estudiando por una parte la posibilidad de establecer diferenciaciones en este sentido, y por la otra examinar si estas eventuales diferenciaciones varían en función de la ocupación efectiva o no de un puesto de trabajo. Este es el objeto del presente trabajo, realizado sobre una población de obreros argentinos. Tres son los objetivos centrales de esta investigación : explorar 1) si los subordinados son conscientes de esta norma de manera global o general o si efectúan diferenciaciones entre sus diversas materializaciones, 2) si, espontáneamente, ellos siguen esta norma en sus diferentes concretizaciones o si actúan de manera selectiva, y 3) si estas actitudes globalizadoras o diferenciadoras varían en función de la ocupación efectiva o no de un puesto de trabajo. 


\section{PROCEDIMIENTO}

Nuestro estudio ha sido efectuado en una población de 180 obreros de la Provincia de Buenos Aires, únicamente masculina, de los cuales la mitad tenía empleo y la otra mitad estaba desocupada (dos grupos de 90 sujetos cada uno).

Los sujetos de cada uno de esos dos grupos fueron invitados a responder a un cuestionario de alineación ya empleado en Francia (Gangloff y Caboux, 2003) constituido por 12 ítems ( $c f$. anexo). Pero, en cada uno de esos dos grupos, se constituyeron tres sub-grupos de treinta sujetos : en el primero los sujetos debían responder a un cuestionario tratando de dar su mejor imagen, en el segundo debían responder de manera espontánea, y en el tercero los sujetos debían responder intentando mostrarse con su peor imagen. La distribución de los sujetos en cada uno de los grupos, figura en la Tabla 1 que sigue.

\begin{tabular}{|l|c|c|c|}
\hline & $\begin{array}{c}\text { Consigna } \\
\text { Sobrenormativa } \\
(\mathbf{N}=6 \mathbf{6 0})\end{array}$ & $\begin{array}{c}\text { Consigna } \\
\text { Honesta } \\
(\mathbf{N}=6 \mathbf{6})\end{array}$ & $\begin{array}{c}\text { Consigna } \\
\text { Contranormativa } \\
(\mathbf{N}=6 \mathbf{6 0})\end{array}$ \\
\hline $\begin{array}{l}\text { Obreros con empleo } \\
(\mathbf{N}=90)\end{array}$ & $\mathrm{N}=30$ & $\mathrm{~N}=30$ & $\mathrm{~N}=30$ \\
\hline $\begin{array}{l}\text { Obreros desempleados } \\
(\mathbf{N}=90)\end{array}$ & $\mathrm{N}=30$ & $\mathrm{~N}=30$ & $\mathrm{~N}=30$ \\
\hline
\end{tabular}

Tabla 1 : distribución de obreros en función a su situación (con empleo / sin empleo) y de la consigna.

Las consignas transmitidas a los sujetos fueron las siguientes:

- Consigna sobrenormativa (CS):

"A continuación, le presentamos un cuestionario que incluye doce afirmaciones.

Le pedimos que lo complete como si se tratara de una planilla que se adjunta a su C.V., que va a ser presentada a un reclutador que decidirá si lo selecciona o no para ocupar un puesto de obrero en la empresa. Le solicitamos que indique si está de acuerdo o no con lo que dice cada una de las afirmaciones marcando con una cruz en el casillero «De acuerdo» o «En desacuerdo».

Finalmente, le pedimos que al responder trate de dar su mejor imagen como si se tratara de caer bien al reclutador y ser as, contratado".

- Consigna honesta $(\mathrm{CH})$ :

"A continuación, le presentamos un cuestionario que incluye doce afirmaciones.

Le pedimos que lo complete como si se tratara de una planilla que se adjunta con su C.V., que va a ser presentada a un reclutador que decidirá si lo selecciona o no para ocupar un puesto de obrero en la empresa. Le solicitamos que indique si está de acuerdo o no con lo que dice cada una de las afirmaciones marcando con una cruz en el casillero «De acuerdo»o «En desacuerdo».

Finalmente, le pedimos que responda de la manera más honesta posible".

- Consigna contranormativa (CC):

"A continuación, le presentamos un cuestionario que incluye doce afirmaciones. 
Le pedimos que lo complete como si se tratara de una planilla que se adjunta a su C.V., que va a ser presentada a un reclutador que decidirá si lo selecciona o no para ocupar un puesto de obrero en la empresa. Le solicitamos que indique si está de acuerdo o no con lo que dice cada una de las afirmaciones marcando con una cruz en el casillero «De acuerdo»o «En desacuerdo».

Finalmente, le pedimos que trate de dar su peor imagen como si se tratara de no caer bien al reclutador, de modo de no ser contratado".

\section{RESULTADOS}

\subsection{Diferencias entre los ítems dentro del grupo}

Nuestro primer análisis ha consistido en calcular las tasas de acuerdo/desacuerdo obtenidas en cada uno de los ítems, para examinar luego las similitudes (versus las diferencias) entre estas proporciones, comparando los ítems de a pares. Estadísticamente hablando, hemos utilizado el test de diferencia de proporciones de McNemar para datos apareados ${ }^{4}$.

De la lectura de las tablas 2 a 7, surge que sobre 66 diferencias significativas posibles, se tienen 22 que corresponden al grupo SOC, 29 al grupo SOS, 26 al grupo HOC, 19 al grupo HOS, 4 al grupo COC y 18 al grupo COS. Esto significa, que como habíamos establecido en nuestra hipótesis de trabajo, existe una diferenciación en las respuestas según los ítems, difererenciación que varía en función de la consigna y de la condición frente al empleo.

Particularmente, es digna de remarcar la importancia de la variable empleo dentro de la consigna contranormativa, con prácticamente cinco veces más diferencias significativas entreítems en el grupo sin empleo (COS) en comparación al grupo con empleo (COC).

$\mathrm{Si}$ tenemos en cuenta que cada ítem puede conducir a 11 diferencias significativas, encontramos que los ítems que difieren significativamente con más de la mitad son los siguientes : dentro del grupo SOC los ítems 7 y 10, dentro del grupo SOS los ítems 8, 10, 3 y 12, dentro del grupo HOC les ítems 10, 12 y 7, dentro del grupo HOS los ítems 10 y 2, y dentro del grupo COS los ítems 8 y 10 .

Esto significa que el ítem que se destaca más frecuentemente de los otros, cuaquiera sea la consigna y la condición frente al empleo, es el ítem 10 (que corresponde a la aceptación del control de los métodos de trabajo y que conduce sistemáticamente a una respuesta de acuerdo, a excepción del grupo COC).

Conviene, sin embargo, investigar un poco más y preguntarse si la variación del número de las diferencias observadas significa o no una polarización de las respuestas en relación a la proporción media teórica. Esta cuestión se aborda en el punto 2.

\footnotetext{
${ }^{4}$ En la aplicación de este test es necesario considerar, no sólo la proporción de individuos que formularon su acuerdo a ambos ítems, sino también la proporción de individuos que formularon su acuerdo a una de las cuestiones pero no a la otra. En consecuencia, dos cuestiones pueden llegar a tener la misma proporción de acuerdos sin que por eso, ambas se diferencien de una tercera cuestión (así, por ej., en la tabla 2, las preguntas 6 y 11 presentan proporciones idénticas, pero sólo el ítem 6 llega a una diferencia significativa en relación al 10).
} 


\begin{tabular}{|l|l|l|l|l|l|l|l|l|l|l|c|c|}
\hline \multicolumn{1}{|c|}{ SOC } & R1 & R2 & R3 & R4 & R5 & R6 & R7 & R8 & R9 & R10 & R11 & R12 \\
\hline R1 $(26 ; 87 \%)$ & & & 0,01 & & & & & & .022 & & & .013 \\
\hline R2 $(27 ; 90 \%)$ & & & 0,01 & & .022 & & & 0,01 & 0,01 & & & 0,01 \\
\hline R3 $(13 ; 43 \%)$ & & & & .022 & & & 0,01 & & & 0,01 & & \\
\hline R4 $(22 ; 73 \%)$ & & & & & & & & & & & & \\
\hline R5 $(18 ; 60 \%)$ & & & & & & 0,01 & & & 0,01 & & \\
\hline R6 $(21 ; 70 \%)$ & & & & & & & .039 & & & .039 & & \\
\hline R7 $(28 ; 93 \%)$ & & & & & & & & 0,01 & 0,01 & & .039 & 0,01 \\
\hline R8 $(19 ; 63 \%)$ & & & & & & & & & & .012 & & \\
\hline R9 $(17 ; 57 \%)$ & & & & & & & & & & 0,01 & & \\
\hline R10 $(28 ; 93 \%)$ & & & & & & & & & & & & 0,01 \\
\hline R11 $(21 ; 70 \%)$ & & & & & & & & & & & & \\
\hline R12 $(15 ; 50 \%)$
\end{tabular}

Tabla 2: valores de los $\mathrm{p}$ obtenidos en caso de diferencias significativas (i.e. 0,05$)$ entre las respuestas para los 12 ítems del cuestionario realizado a los obreros con empleo, bajo la consigna sobrenormativa (Sobrenormativa Obreros Con empleo). Se agregan las cantidades de acuerdos y porcentajes obtenidos para cada respuesta.

\begin{tabular}{|l|l|l|l|l|l|l|l|l|l|l|c|c|}
\hline \multicolumn{1}{|c|}{ SOS } & R1 & R2 & R3 & R4 & R5 & R6 & R7 & R8 & R9 & R10 & R11 & R12 \\
\hline R1 $(24 ; 80 \%)$ & & & 0,01 & & & & & 0,01 & & & & 0,01 \\
\hline R2 $(27 ; 90 \%)$ & & & 0,01 & & .012 & & & 0,01 & & & .039 & 0,01 \\
\hline R3 $(12 ; 40 \%)$ & & & & 0,01 & & & .021 & & 0,01 & 0,01 & & \\
\hline R4 $(26 ; 87 \%)$ & & & & & .039 & & & 0,01 & & & .016 & 0,01 \\
\hline R5 $(18 ; 60 \%)$ & & & & & & & & .039 & & 0,01 & & \\
\hline R6 $(20 ; 67 \%)$ & & & & & & & & 0,01 & & .012 & & \\
\hline R7 $(22 ; 73 \%)$ & & & & & & & & 0,01 & & .039 & & .022 \\
\hline R8 $(10 ; 33 \%)$ & & & & & & & & & 0,01 & 0,01 & .022 & \\
\hline R9 $(24 ; 80 \%)$ & & & & & & & & & & & & 0,01 \\
\hline R10 $(29 ; 97 \%)$ & & & & & & & & & & & 0,01 & 0,01 \\
\hline R11 $(19 ; 63 \%)$ & & & & & & & & & & & & \\
\hline R12 $(13 ; 43 \%)$ & & & & & & & & & & \\
\hline
\end{tabular}

Tabla 3: valores de los $\mathrm{p}$ obtenidos en caso de diferencias significativas (i.e. 0,05$)$ entre las respuestas para los 12 ítems del cuestionario realizado a los obreros sin empleo, bajo la consigna sobrenormativa (Sobrenormativa Obreros Sin empleo). Se agregan las cantidades de acuerdos y porcentajes obtenidos para cada respuesta. 


\begin{tabular}{|c|c|c|c|c|c|c|c|c|c|c|c|c|}
\hline HOC & R1 & $\mathbf{R 2}$ & $\mathbf{R 3}$ & R4 & R5 & R6 & R7 & R8 & R9 & R10 & R11 & R12 \\
\hline R1 $(23 ; 77 \%)$ & & & & & .035 & & & .035 & .021 & & & 0,01 \\
\hline R2 $(25 ; 83 \%)$ & & & & & 0,01 & & & 0,01 & .013 & & & 0,01 \\
\hline $\mathbf{R 3}(18 ; 60 \%)$ & & & & & & & .039 & & & .012 & & .013 \\
\hline R4 $(18 ; 60 \%)$ & & & & & & & .039 & & & .012 & & 0,01 \\
\hline R5 $(14 ; 47 \%)$ & & & & & & & 0,01 & & & 0,01 & & \\
\hline R6 $(20 ; 67 \%)$ & & & & & & & & & & .039 & & 0,01 \\
\hline R7 $(26 ; 87 \%)$ & & & & & & & & 0,01 & 0,01 & & & 0,01 \\
\hline R8 $(14 ; 47 \%)$ & & & & & & & & & & 0,01 & & \\
\hline R9 $(15 ; 50 \%)$ & & & & & & & & & & 0,01 & & \\
\hline $\mathbf{R 1 0}(27 ; 90 \%)$ & & & & & & & & & & & 0,01 & 0,01 \\
\hline R11 $(19 ; 63 \%)$ & & & & & & & & & & & & 0,01 \\
\hline R12 $(8 ; 27 \%)$ & & & & & & & & & & & & \\
\hline
\end{tabular}

Tabla 4: valores de los $\mathrm{p}$ obtenidos en caso de diferencias significativas (i.e. 0,05) entre las respuestas para los 12 ítems del cuestionario realizado a los obreros con empleo, bajo la consigna honesta (Honesta Obreros Con empleo). Se agregan las cantidades de acuerdos y porcentajes obtenidos para cada respuesta.

\begin{tabular}{|c|c|c|c|c|c|c|c|c|c|c|c|c|}
\hline HOS & R1 & $\mathbf{R 2}$ & $\mathbf{R 3}$ & R4 & $\mathbf{R 5}$ & R6 & R7 & R8 & R9 & R10 & R11 & R12 \\
\hline R1 $(20 ; 67 \%)$ & & & & & & & & .031 & & & & \\
\hline R2 $(24 ; 80 \%)$ & & & .013 & & .013 & .039 & & 0,01 & .021 & & & .013 \\
\hline R3 $(14 ; 47 \%)$ & & & & & & & .035 & & & .013 & & \\
\hline R4 $(20 ; 67 \%)$ & & & & & & & & .021 & & & & \\
\hline R5 $(14 ; 47 \%)$ & & & & & & & .035 & & & .013 & & \\
\hline R6 $(16 ; 53 \%)$ & & & & & & & & & & .039 & & \\
\hline R7 $(23 ; 77 \%)$ & & & & & & & & 0,01 & .022 & & & .035 \\
\hline R8 $(10 ; 33 \%)$ & & & & & & & & & & 0,01 & & \\
\hline R9 $(14 ; 47 \%)$ & & & & & & & & & & .013 & & \\
\hline $\mathbf{R 1 0}(24 ; 80 \%)$ & & & & & & & & & & & & 0,01 \\
\hline $\mathbf{R 1 1}(17 ; 57 \%)$ & & & & & & & & & & & & \\
\hline $\mathbf{R 1 2}(14 ; 47 \%)$ & & & & & & & & & & & & \\
\hline
\end{tabular}

Tabla 5: valores de los $\mathrm{p}$ obtenidos en caso de diferencias significativas (i.e. 0,05) entre las respuestas para los 12 ítems del cuestionario realizado a los obreros sin empleo, bajo la consigna honesta (Honesta Obreros Sin empleo). Se agregan las cantidades de acuerdos y porcentajes obtenidos para cada respuesta. 


\begin{tabular}{|l|l|l|l|l|l|l|l|l|l|l|l|l|}
\hline \multicolumn{1}{|c|}{ COC } & R1 & R2 & R3 & R4 & R5 & R6 & R7 & R8 & R9 & R10 & R11 & R12 \\
\hline R1 $(11 ; 37 \%)$ & & & & & & & & & & & \\
\hline R2 $(6 ; 20 \%)$ & & 0,01 & & & & & & & & & \\
\hline R3 $(17 ; 57 \%)$ & & & & & .012 & & .021 & 0,01 & & & \\
\hline R4 $(14 ; 47 \%)$ & & & & & & & & & & & & \\
\hline R5 $(11 ; 37 \%)$ & R6 $(8 ; 27 \%)$ \\
\hline R7 $(11 ; 37 \%)$ & & & & & & & & & & & & \\
\hline R8 $(7 ; 23 \%)$ & & & & & & & & & & & \\
\hline R9 $(8 ; 27 \%)$ & & & & & & & & & & & \\
\hline R10 $(11 ; 37 \%)$ & & & & & & & & & & & \\
\hline R11 $(10 ; 33 \%)$ & & & & & & & & & & & \\
\hline R12 $(10 ; 33 \%)$ & & & & & & & & & & & \\
\hline
\end{tabular}

Tabla 6: valores de los $\mathrm{p}$ obtenidos en caso de diferencias significativas (i.e. 0,05) entre las respuestas para los 12 ítems del cuestionario realizado a los obreros con empleo, bajo la consigna contranormativa (Contranormativa Obreros Con empleo). Se agregan las cantidades de acuerdos y porcentajes obtenidos para cada respuesta.

\begin{tabular}{|c|c|c|c|c|c|c|c|c|c|c|c|c|}
\hline COS & R1 & R2 & R3 & R4 & R5 & R6 & R7 & R8 & R9 & R10 & R11 & R12 \\
\hline R1 $(15 ; 50 \%)$ & & & & & & & & .021 & & .013 & & \\
\hline R2 $(17 ; 57 \%)$ & & & & & & & & 0,01 & & & & \\
\hline R3 $(12 ; 40 \%)$ & & & & & & & .031 & & & 0,01 & & \\
\hline R4 $(17 ; 57 \%)$ & & & & & & & & .021 & & .021 & & \\
\hline R5 $(13 ; 43 \%)$ & & & & & & & .049 & & & 0,01 & & \\
\hline R6 $(11 ; 37 \%)$ & & & & & & & 0,01 & & & 0,01 & & \\
\hline R7 $(22 ; 73 \%)$ & & & & & & & & 0,01 & & & & .013 \\
\hline $\mathbf{R 8}(7 ; 23 \%)$ & & & & & & & & & .039 & 0,01 & 0,01 & \\
\hline R9 $(15 ; 50 \%)$ & & & & & & & & & & 0,01 & & \\
\hline $\mathbf{R 1 0}(25 ; 83 \%)$ & & & & & & & & & & & & 0,01 \\
\hline $\mathbf{R 1 1}(17 ; 57 \%)$ & & & & & & & & & & & & \\
\hline R12 $(12 ; 40 \%)$ & & & & & & & & & & & & \\
\hline
\end{tabular}

Tabla 7: valores de los $\mathrm{p}$ obtenidos en caso de diferencias significativas (i.e. 0,05) entre las respuestas para los 12 ítems del cuestionario realizado a los obreros sin empleo, bajo la consigna contranormativa (Contranormativa Obreros Sin empleo). Se agregan las cantidades de acuerdos y porcentajes obtenidos para cada respuesta.

\subsection{Diferencias respecto de la proporción media teórica}

Nos hemos preguntado ahora si las respuestas de los sujetos difieren de la proporción media teórica - dicha proporción tiene el valor 0,5 y significa que la mitad de las personas del grupo está de acuerdo y que la otra mitad no lo está - y hemos efectuado esta comparación en base al test exacto de Fisher. Los resultados detallados figuran en la Tabla 8 y se resumen en la Tabla 9. Algunos hallazgos son dignos de remarcar :

- en el caso de los obreros con empleo, se observan 4 ítems donde las respuestas son superiores a la proporción media teórica, tanto en la consigna neutra u honesta, como en la sobrenormativa (los ítems 1, 2, 7 y 10). Por el contrario, en consigna contranormativa, el ítem 2 (que corresponde 
a la ejecución sistemática de órdenes) conduce a respuestas obedientes inferiores a la proporción media teórica. Esto significa que el ítem 2, en esta población, produce una fuerte variación entre consignas, y por tanto, constituye el ítem con más fuerte clarividencia normativa.

- en el caso de los obreros sin empleo, se obtienen respuestas superiores a la proporción media teórica, nuevamente en los ítems 2 y 10 (el 10 alude a la aceptación del control de los métodos de trabajo), tanto en la consigna sobrenormativa como en la consigna honesta.

\begin{tabular}{|c|c|c|c|c|c|c|c|c|c|c|c|c|}
\hline & 1 & 2 & 3 & 4 & 5 & 6 & 7 & 8 & 9 & 10 & 11 & 12 \\
\hline \multirow[t]{2}{*}{ SOC } & $\begin{array}{c}26 \\
(87 \%) \\
\end{array}$ & $\begin{array}{c}27 \\
(90 \%)\end{array}$ & $\begin{array}{c}13 \\
(43 \%)\end{array}$ & $\begin{array}{c}22 \\
(73 \%)\end{array}$ & $\begin{array}{c}18 \\
(60 \%)\end{array}$ & $\begin{array}{c}21 \\
(70 \%)\end{array}$ & $\begin{array}{c}28 \\
(93 \%)\end{array}$ & $\begin{array}{c}19 \\
(63 \%)\end{array}$ & $\begin{array}{c}17 \\
(57 \%)\end{array}$ & $\begin{array}{c}28 \\
(93 \%)\end{array}$ & $\begin{array}{c}21 \\
(70 \%)\end{array}$ & $\begin{array}{c}15 \\
(50 \%)\end{array}$ \\
\hline & 0,01 & 0,01 & & & & & 0,01 & & & 0,01 & & \\
\hline \multirow[t]{2}{*}{ SOS } & $\begin{array}{c}24 \\
(80 \%) \\
\end{array}$ & $\begin{array}{c}27 \\
(90 \%) \\
\end{array}$ & $\begin{array}{c}12 \\
(40 \%)\end{array}$ & $\begin{array}{c}26 \\
(87 \%) \\
\end{array}$ & $\begin{array}{c}18 \\
(60 \%) \\
\end{array}$ & $\begin{array}{c}20 \\
(67 \%) \\
\end{array}$ & $\begin{array}{c}22 \\
(73 \%)\end{array}$ & $\begin{array}{c}10 \\
(33 \%)\end{array}$ & $\begin{array}{c}24 \\
(80 \%) \\
\end{array}$ & $\begin{array}{c}29 \\
(97 \%) \\
\end{array}$ & $\begin{array}{c}19 \\
(63 \%) \\
\end{array}$ & $\begin{array}{c}13 \\
(43 \%) \\
\end{array}$ \\
\hline & 0,01 & 0,01 & & 0,01 & & & & & 0,01 & 0,01 & & \\
\hline \multirow[t]{2}{*}{ HOC } & $\begin{array}{c}23 \\
(77 \%)\end{array}$ & $\begin{array}{c}25 \\
(83 \%)\end{array}$ & $\begin{array}{c}18 \\
(60 \%)\end{array}$ & $\begin{array}{c}18 \\
(60 \%) \\
\end{array}$ & $\begin{array}{c}14 \\
(47 \%)\end{array}$ & $\begin{array}{c}20 \\
(67 \%) \\
\end{array}$ & $\begin{array}{c}26 \\
(87 \%)\end{array}$ & $\begin{array}{c}14 \\
(47 \%)\end{array}$ & $\begin{array}{c}15 \\
(50 \%)\end{array}$ & $\begin{array}{c}27 \\
(90 \%)\end{array}$ & $\begin{array}{c}19 \\
(63 \%)\end{array}$ & $\begin{array}{c}8 \\
(27 \%)\end{array}$ \\
\hline & 0,03 & 0,01 & & & & & 0,01 & & & 0,01 & & \\
\hline \multirow[t]{2}{*}{ HOS } & $\begin{array}{c}20 \\
(67 \%)\end{array}$ & $\begin{array}{c}24 \\
(80 \%)\end{array}$ & $\begin{array}{c}14 \\
(47 \%) \\
\end{array}$ & $\begin{array}{c}20 \\
(67 \%) \\
\end{array}$ & $\begin{array}{c}14 \\
(47 \%) \\
\end{array}$ & $\begin{array}{c}16 \\
(53 \%) \\
\end{array}$ & $\begin{array}{c}23 \\
(77 \%)\end{array}$ & $\begin{array}{c}10 \\
(33 \%)\end{array}$ & $\begin{array}{c}14 \\
(47 \%) \\
\end{array}$ & $\begin{array}{c}24 \\
(80 \%)\end{array}$ & $\begin{array}{c}17 \\
(57 \%) \\
\end{array}$ & $\begin{array}{c}14 \\
(47 \%) \\
\end{array}$ \\
\hline & & 0,01 & & & & & 0,03 & & & 0,01 & & \\
\hline \multirow[t]{2}{*}{$\mathrm{COC}$} & $\begin{array}{c}11 \\
(37 \%) \\
\end{array}$ & $\begin{array}{c}6 \\
(20 \%) \\
\end{array}$ & $\begin{array}{c}17 \\
(57 \%) \\
\end{array}$ & $\begin{array}{c}14 \\
(47 \%)\end{array}$ & $\begin{array}{c}11 \\
(37 \%)\end{array}$ & $\begin{array}{c}8 \\
(27 \%) \\
\end{array}$ & $\begin{array}{c}11 \\
(37 \%)\end{array}$ & $\begin{array}{c}7 \\
(23 \%)\end{array}$ & $\begin{array}{c}8 \\
(27 \%)\end{array}$ & $\begin{array}{c}11 \\
(37 \%)\end{array}$ & $\begin{array}{c}10 \\
(33 \%) \\
\end{array}$ & $\begin{array}{c}10 \\
(33 \%) \\
\end{array}$ \\
\hline & & 0.01 & & & & & & 0.03 & & & & \\
\hline \multirow[t]{2}{*}{ COS } & $\begin{array}{c}15 \\
(50 \%) \\
\end{array}$ & $\begin{array}{c}17 \\
(57 \%) \\
\end{array}$ & $\begin{array}{c}12 \\
(40 \%) \\
\end{array}$ & $\begin{array}{c}17 \\
(57 \%)\end{array}$ & $\begin{array}{c}13 \\
(43 \%) \\
\end{array}$ & $\begin{array}{c}11 \\
(37 \%)\end{array}$ & $\begin{array}{c}22 \\
(73 \%) \\
\end{array}$ & $\begin{array}{c}7 \\
(23 \%) \\
\end{array}$ & $\begin{array}{c}15 \\
(50 \%) \\
\end{array}$ & $\begin{array}{c}25 \\
(83 \%) \\
\end{array}$ & $\begin{array}{c}17 \\
(57 \%)\end{array}$ & $\begin{array}{c}12 \\
(40 \%) \\
\end{array}$ \\
\hline & & & & & & & & 0,03 & & 0,01 & & \\
\hline
\end{tabular}

Tabla 8: número de acuerdos obtenidos por ítem. Se incluye el porcentaje entre paréntesis, para cada uno de los 6 grupos de obreros. En negrita se remarca el p-valor en relación a la proporción media teórica en caso de diferencias significativas.

\begin{tabular}{|ll|c|c|}
\hline \multirow{4}{*}{ Sobrenormativa } & \multicolumn{2}{c|}{ Obreros } \\
\cline { 2 - 4 } & Con empleo & Sin empleo \\
$1,2,7,10$ & $1,2,4,9,10$ \\
\hline \multirow{4}{*}{ Conesta } & Superior a la proporción media teórica & & \\
\cline { 2 - 4 } & $\begin{array}{l}\text { Inferior a la proporción media teórica } \\
\text { Superior a la proporción media teórica }\end{array}$ & $1,2,7,10$ & $2,7,10$ \\
\cline { 2 - 4 } & $\begin{array}{l}\text { Inferior a la proporción media teórica } \\
\text { Superior a la proporción media teórica }\end{array}$ & & 10 \\
\cline { 2 - 4 } & Inferior a la proporción media teórica & 2,8 & 8 \\
\hline
\end{tabular}

Tabla 9: número de ítems para los cuales los obreros expresan un acuerdo significativamente superior o inferior a la proporción media teórica 


\subsection{Diferencias entre grupos por ítem}

Nuestro próximo interrogante es acerca de las eventuales diferencias de respuestas entre grupos, examen realizado en base al test exacto de Fisher.

La tabla 10 muestra que son los ítems 2 (ejecución sistemática de órdenes) y 7 (evitación de crear problemas) los que conducen a las variaciones más frecuentes.

La variable consigna permite constatar, entre la consigna sobrenormativa y la contranormativa, 9 diferencias significativas (sobre 12 posibles) para los obreros con empleo (SOC/COC) y 5 para los obreros sin empleo (SOS/COS). Los obreros sin empleo manifiestan menos clarividencia en las diferentes materializaciones de la norma de obediencia.

Si se mantiene el foco en la condición frente al empleo (con empleo o sin empleo) se observa que respecto de la consigna sobrenormativa (SOC/SOS), existen respuestas diferentes para los ítemas 7 y 8 , con tasas de aprobación más importantes para los que tienen empleo (el ítem 8 corresponde a la legitimidad del jefe), y que respecto de la consigna contranormativa, se registran respecto de los ítems 2, 7 y 10, porcentajes de acuerdo más elevados para los desocupados (el ítem 10 refiere al control de los métodos de trabajo).

\begin{tabular}{|c|c|c|c|c|c|c|c|c|c|c|c|c|c|}
\hline SOC & SOS & 1 & 2 & 3 & 4 & 5 & 6 & $\begin{array}{c}7 \\
0,04 \\
\end{array}$ & $\begin{array}{c}\mathbf{8} \\
0,02\end{array}$ & 9 & 10 & 11 & 12 \\
\hline & HOC & & & & & & & & & & & & \\
\hline SOS & $\begin{array}{l}\mathrm{COC} \\
\mathrm{HOS}\end{array}$ & 0,01 & 0,01 & & 0,04 & & 0,01 & 0,01 & 0,01 & $\begin{array}{c}0,02 \\
0,01\end{array}$ & 0,01 & 0,01 & \\
\hline HOC & $\begin{array}{ll}\text { COS } \\
\text { HOS }\end{array}$ & 0,02 & 0,01 & & 0,01 & & 0,02 & & & 0,01 & & & \\
\hline $\begin{array}{l}\text { HOS } \\
\text { COC }\end{array}$ & $\begin{array}{l}\mathrm{COC} \\
\mathrm{COS} \\
\mathrm{COS}\end{array}$ & 0,01 & $\begin{array}{l}0,01 \\
0,01\end{array}$ & & & & 0,01 & $\begin{array}{l}0,01 \\
0,01\end{array}$ & & & $\begin{array}{l}0,01 \\
0,01\end{array}$ & 0,02 & \\
\hline
\end{tabular}

Tabla 10: valores de los p en caso de diferencias significativas (i.e. cuando son inferiores a 0,05 ) entre los grupos, por ítem (así, entre SOC y SOS, para el ítem 7, la diferencia es significativa con un $\mathrm{p}=0,04)$.

\section{DISCUSION-CONCLUSION}

Las diferencias observadas entre consignas sobrenormativa y contranormativa (es decir frente a la solicitud de dar su mejor o peor imagen) indican que existe clarividencia normativa. Sin embargo, estas diferencias no se registran en la totalidad de los ítems, lo que significa, conforme a nuestras hipótesis, que esta clarividencia es selectiva. En especial, es digno de destacar ( $c f$. tabla 10) que los ítems 3 (la no defensa del propio punto de vista), 5 (la autocensura), y 12 (la renuncia a pedir justificación por las decisiones tomadas), no son objeto de ningún tipo de clarividencia, más allá de cual sea la condición frente al empleo.

En este sentido, se observa que, espontáneamente, los obreros que hemos interrogado no responden de la misma manera a cada uno de los ítems, con 26 diferencias inter-ítems en el grupo HOC y 19 en el grupo HOS ( $c f$. tablas 4 y 5). 
Hemos constatado asimismo que estos resultados varían según que interroguemos a obreros dotados de empleo o sin él, confirmándose así el efecto de la variable empleo. En particular, hemos observado dentro de la consigna contranormativa que los obreros con empleo producen prácticamente cinco veces menos diferenciaciones inter-ítems que los obreros sin empleo ( $c f$. tablas 6 y 7 ).

Esta menor diferenciación de obreros con empleo pone de manifiesto el rol normalizador que las organizaciones llevan a cabo, especialmente en torno a las conductas contranormativas, es decir a nivel de las conductas que conviene evitar. Tal resultado confirma ciertas observaciones según las cuales la inserción laboral/profesional tendría, al menos en nuestras sociedades industrializadas, un objetivo no solamente económico, sino también político, de perennización de las estructuras de poder, vía la conformidad social. Por otra parte, y desde un plano metaanalítico, esto nos recuerda que la psicología social de las organizaciones posee ramificaciones y áreas de intersección con otras disciplinas de las ciencias humanas, ramificaciones y intersecciones que invitan a una aproximación sistémica.

\section{BIBLIOGRAFIA}

Caprara G.V., Barbaranelli C., et Borgogni L. (1997). Alter ego; les 5 facteurs fondamentaux de la personnalité. Paris: E.A.P.

Gangloff B. (2000). La saturation normative des tests dits de personnalité. Communication au $27^{\text {ème }}$ Congrès International de Psychologie, Stockholm (Suède).

Gangloff B. (2002). L'internalité et l'allégeance considérées comme des normes: une revue. Les Cahiers de Psychologie Politique, $\mathrm{n}^{\circ} 2$.

Gangloff B. et Caboux N. (2003). Conformismo a la norma de alineación y reacciones a las injusticias profesionales. $29^{\text {ème }}$ Congreso Interamericano de Psicología. Lima, Perú.

Gangloff B. et Huet M. (2004). Poids respectif du gros cinq et de l'allégeant en situation de recrutement. Communication au 13ème Congrès International de Psychologie du Travail et de Organisations, Bologne (Italie).

Gangloff B., Mayoral L., et Duringer L. (2005). Los asalariados que prefieren los jefes argentinos: influencia del nivel de alineacion y del género, $30^{\text {ème }}$ Congrès de la Société Interaméricaine de Psychologie, Buenos Aires (Argentine).

Gangloff B., Mayoral L., et Quiroga F. (2005). La percepcion de los obreros argentinos en cuanto al nivel de alineacion conveniente para ser reclutados, $30^{\text {ème }}$ Congrès de la Société Interaméricaine de Psychologie, Buenos Aires (Argentine).

Py J. et Somat A. (1991). Normativité, conformité et clairvoyance: leurs effets sur le jugement évaluatif dans un contexte scolaire. In: J-L. Beauvois, R-V. Joule et J-M. Monteil (Eds), Perpectives cognitives et conduites sociales. Cousset: Del Val, vol. 3, 167-193.

Rolland J-P. (1994). Désirabilité sociale de marqueurs des dimensions de ma personnalité du modèle en 5 facteurs. Revue Européenne de Psychologie Apliquée, 44(1), 65-71. 


\section{Anexo : el cuestionario utilizado}

Este cuestionario comprende 9 ítems sobre las manifestaciones de la obediencia (ítems 1, 2, 3, 4, 5, 7, 9, 10 y 12) y 3 ítems sobre las razones de la obediencia (ítems 6, 8, 11). Se indica entre paréntesis y en negrita la dimensión presente en cada uno de los ítems. (Esto no figuraba en el cuestionario presentado a los sujetos)

1/ En el trabajo, me parece normal pedir sistemáticamente el acuerdo de mi jefe antes de tomar una decisión (abandono de toda autonomía decisoria).

2/ En mi trabajo, me parece normal ejecutar todas las órdenes que me da mi superior (ejecución sistemática de órdenes).

3/ Con mi jefe, intento raramente defender mis ideas (no defensa del propio punto de vista).

4/ Cuando la dirección me comunica sus decisiones, es raro que yo discuta abiertamente (no cuestionamiento a las decisiones).

5/ Evito hacer observaciones a mi superior, incluso si, en algunas ocasiones, se lo merece (autocensura).

6/ Encuentro normal aceptar todas las decisiones de mis superiores si yo quiero subir en la escala jerárquica. (funcionalidad de la aceptación de decisiones de la autoridad en términos de crecimiento laboral).

7/ Cualesquiera sean las condiciones de trabajo, encuentro normal evitar crear problemas (evitación de crear problemas).

8/ Acepto más fácilmente un tono autoritario cuando este viene de mi jefe (reconocimiento de la legitimitad del jefe).

9/ En todas las circunstancias, encuentro normal que la opinión de mi superior sea más importante que la mía (aceptación de la predominancia del punto de vista del jefe)

10/ Encuentro normal que mi superior quiera verificar, no importa en qué momento, si yo respeto los métodos de trabajo (aceptación del control de los metodos de trabajo)

11/ Me digo con frecuencia que mi patrón es el mejor ubicado para saber qué es lo bueno para su empresa (reconocimiento de la expertise del jefe)

12/ Encuentro normal que mi patrón no justifique sus decisiones ante mí, incluso si ellas me conciernen en forma directa (renuncia a pedir justificación por las decisiones).

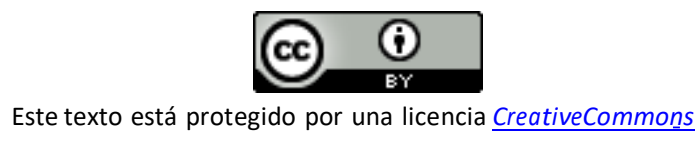

$\underline{4.0}$.

Usted es libre para Compartir - copiar y redistribuir el material en cualquier medio o formato - y Adaptar el documen- to -remezclar, transformar y crear a partir del material- para cualquier propósito, incluso comercialmente, siempre que cumpla la condición de:

Atribución: Usted debe reconocer el crédito de una obra de manera adecuada, proporcionar un enlace a la licencia, e in- dicar si se han realizado cambios. Puede hacerlo en cualquier forma razonable, pero no de forma tal que sugiera que tie- ne el apoyo del licenciante o lo recibe por el uso que hace. 